\title{
Multicomponent fractional quantum Hall effect in graphene
}

\author{
C. R. Dean ${ }^{1,2 \dagger}$, A. F. Young ${ }^{3 \dagger}$, P. Cadden-Zimansky ${ }^{3,4}$, L. Wang ${ }^{2}$, H. Ren ${ }^{3}$, K. Watanabe ${ }^{5}$, T. Taniguchi ${ }^{5}$, \\ P. $\mathrm{Kim}^{3 \star}$, J. Hone ${ }^{2}$ and K. L. Shepard ${ }^{1}$
}

The fractional quantum Hall effect ${ }^{1-4}$ (FQHE) in an electron gas with multiple internal degrees of freedom provides a model system to study the interplay between symmetry breaking and emergent topological order ${ }^{5}$. In graphene, the structure of the honeycomb lattice endows the electron wavefunctions with an additional quantum number, termed valley isospin, which, combined with the usual electron spin, yields fourfold degenerate Landau levels (LLs; refs 6,7). This additional symmetry modifies the FQHE and is conjectured to produce new incompressible ground states in graphene ${ }^{8-17}$. Here we report multiterminal measurements of the FQHE in highmobility graphene devices fabricated on hexagonal boron nitride substrates ${ }^{18}$. The measured energy gaps are large, particularly in the second Landau level, where they are up to 10 times larger than those reported in the cleanest conventional systems. In the lowest Landau level the hierarchy of FQH states reflects the additional valley degeneracy.

The anomalous quantum Hall effect in graphene $e^{6,7}$, in which Hall plateaux appear at $v= \pm 4(n+1 / 2)$ for Landau level index $n=0,1,2 \ldots$, can be understood using a theory of non-interacting, massless Dirac fermions in which the four-fold level degeneracy reflects the two spin and two valley degrees of freedom, and the $1 / 2$ offset in the ladder of QHE states relates to the non-zero Berry phase acquired by Dirac fermions in a magnetic field. Each LL is approximately $\mathrm{SU}(4)$ symmetric, as symmetry breaking effects, such as Zeeman coupling and contact interactions, are weak at experimentally realizable magnetic fields. Even at $B=35 \mathrm{~T}$, the ratio between the Zeeman and Coulomb energies is $E_{\mathrm{Z}} / E_{\mathrm{C}} \sim 0.01 \epsilon$ (where $\epsilon$ is the dielectric constant), and lattice scale interactions are only $a / l_{\mathrm{B}} \sim 0.06$ (where $a$ is the lattice constant and $l_{\mathrm{B}}=\sqrt{\hbar / e B}$ is the magnetic length). Exchange interactions can favour ground states with an $\mathrm{SU}(4)$ ferromagnetic polarization ${ }^{9,10}$, yielding the observed $^{18-21}$ IQHE at all integer $v$ in the cleanest samples. At fractional filling, the expanded symmetry of the LLs opens up the possibility of observing novel many-body states that have no analogue in one- and two-component systems ${ }^{12,14}$.

In this Letter we report on measurements of the FQHE in a single-layer graphene sample fabricated on a hexagonal boron nitride (h-BN) substrate (see Methods). Figure la shows the zero-field resistance and corresponding conductivity, acquired at $T \sim 300 \mathrm{mK}$. The Hall mobility at high density is $\sim 30,000 \mathrm{~cm}^{2} \mathrm{Vs}^{-1}$; the charged-impurity mobility, which dominates at low density, is in excess of $100,000 \mathrm{~cm}^{2} \mathrm{Vs}^{-1}$, as determined by fitting straight lines to the linear portion of the conductivity; the charge inhomogeneity, estimated from the resistivity peak width at the charge neutrality a
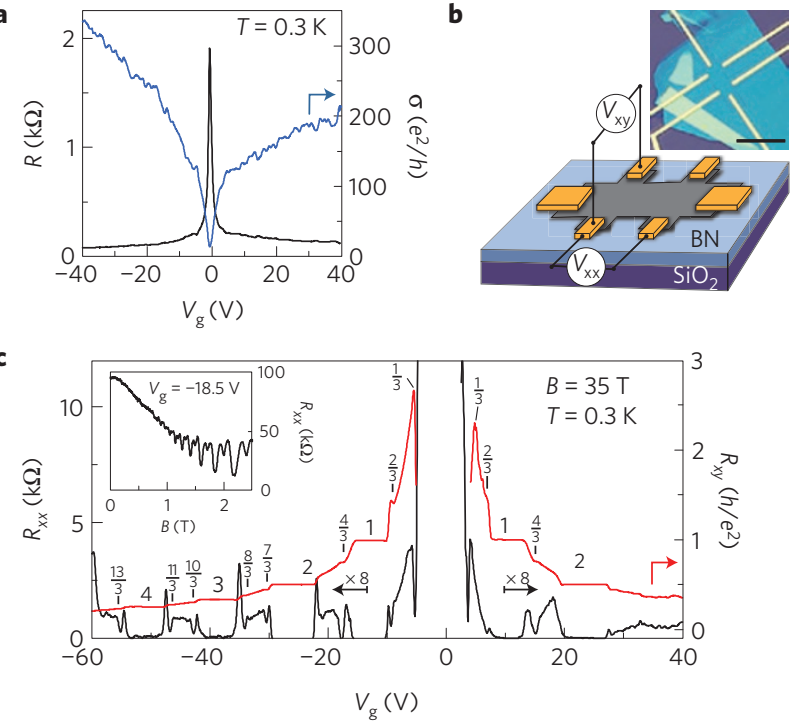

Figure 1 | Magnetotransport. a, Resistance and conductivity measured at zero magnetic field. $\mathbf{b}$, Schematic of the etched Hall bar device. Inset:

Optical image; scale bar is $5 \mu \mathrm{m}$. c, Magnetoresistance (left axis) and Hall resistance (right axis) versus gate voltage acquired at $B=35 \mathrm{~T}$. Inset shows $\mathrm{SdH}$ oscillations at $V_{g}=-18.5 \mathrm{~V}$.

point (CNP), is of the order $10^{10} \mathrm{~cm}^{-2}$. All three metrics indicate this sample to be of exceptionally high quality and are consistent with previous measurements of similar graphene/h-BN devices ${ }^{18}$.

Longitudinal $\left(R_{x x}\right)$ and Hall $\left(R_{x y}\right)$ magnetoresistance measurements acquired by varying the gate voltage at a fixed $B$ field of $35 \mathrm{~T}$ are shown in Fig. 1c. Quantized Hall plateaux and magnetoresistance zeros appear at all accessible integer fillings in both the $n=0$ and $n=1$ LLs. Signatures of symmetry-breaking appear in the Shubnikov-de Haas ( $\mathrm{SdH}$ ) oscillations at fields as low as $1 \mathrm{~T}$ (inset of Fig. 1c), with full breaking of the four-fold degeneracy observed at fields less than $5 \mathrm{~T}$. The most remarkable feature of this sample is the appearance of the FQHE at $35 \mathrm{~T}$ throughout the accessible density range (labelled with arrows in Fig. 1c). In the remainder of this Letter, we focus our attention on the FQHE states, with analysis of the integer QHE (IQHE) states to be given elsewhere.

In Fig. 2a,b, detailed plots of the $n=0$ and $n=1$ LLs are given. For clarity, the Hall conductivity, calculated from the tensor relation $\sigma_{x y}=R_{x y} /\left(R_{x y}^{2}+(w / l) R_{x x}^{2}\right)$, is shown, where $w / l$ is the aspect ratio

\footnotetext{
${ }^{1}$ Department of Electrical Engineering, Columbia University, New York, New York 10027, USA, 2 Department of Mechanical Engineering, Columbia University, New York, New York 10027, USA, ${ }^{3}$ Department of Physics, Columbia University, New York, New York 10027, USA, ${ }^{4}$ National High Magnetic Field Laboratory, Tallahassee, Florida 32310, USA, ${ }^{5}$ Advanced Materials Laboratory, National Institute for Materials Science, 1-1 Namiki, Tsukuba 305-0044, Japan. †These authors contributed equally to this work. *e-mail: pk2015@columbia.edu.
} 


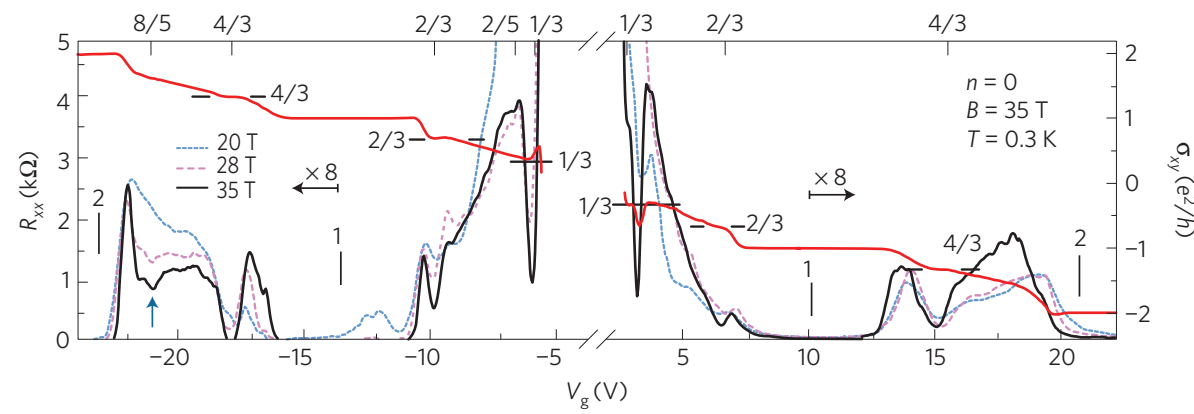

b

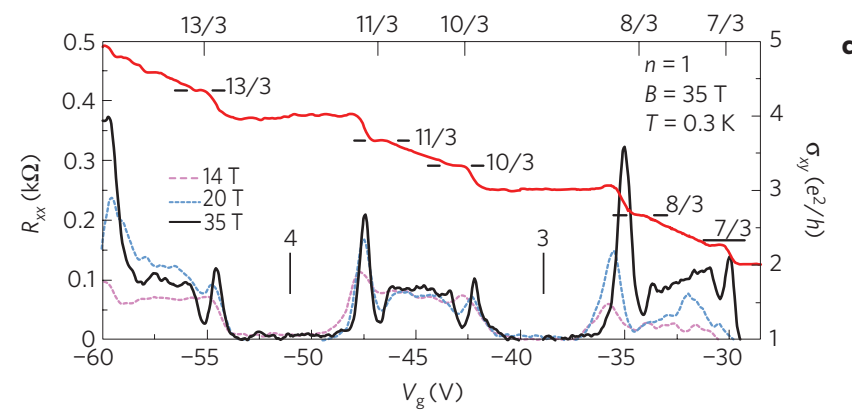

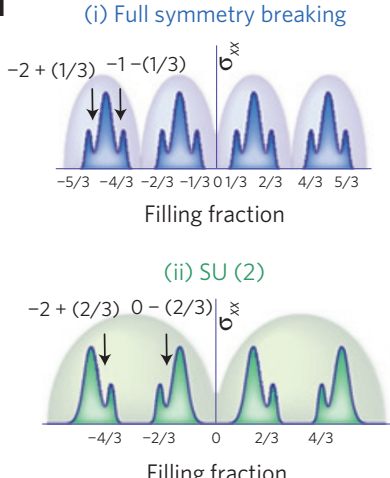

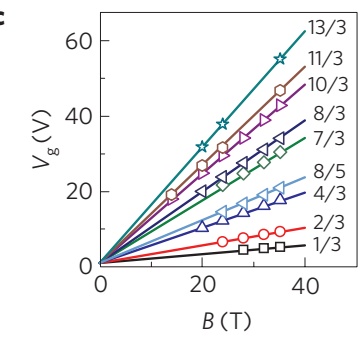

(iii) Observed fractions

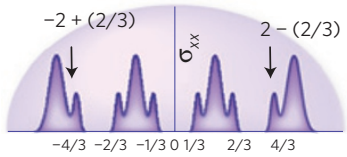

Filling fraction

Figure 2 | Fractional quantum Hall effect. a,b, Magnetoresistance (left axis) and Hall conductivity (right axis) in the $n=0$ and $n=1$ Landau levels at $B=35 \mathrm{~T}$ and $T \sim 0.3 \mathrm{~K}$. c, Fan diagram showing the resistance minima at different magnetic fields for the FQHE states labelled in $\mathbf{a}$ and $\mathbf{b}$. Lines correspond to the positions calculated from the relation $B_{v}=(1 / v) n_{\mathrm{e}} h / e$, where $n_{\mathrm{e}}=C_{\mathrm{g}}\left(\mathrm{V}-\mathrm{V}_{\mathrm{o}}\right) / \mathrm{e}$ is the carrier density and $C_{\mathrm{g}}=1.09 \times 10^{-4} \mathrm{~F} \mathrm{~m}^{-2}$ the capacitive coupling to the doped Si back gate, determined independently from low- $B$ Hall measurements. d, Schematic representation of the FQHE hierarchy observed in our sample. Shown is the expected electron-hole symmetry assuming (i) full lifting of all internal degeneracies due to, for example, coupling to external fields, (ii) full breaking of only one degeneracy, leaving a two-fold degenerate branch, (iii) schematic diagram of the experimental situation. Arrows label particle-hole conjugate pairs in each scenario.

of the Hall bar. We observe the hallmark features of the FQHE, namely quantization of $R_{x y}$ to values of $(1 / v) h / e^{2}$ concomitant with minima in $R_{x x}$, at fractional filling factors $v=1 / 3,2 / 3$ and $4 / 3$ in the $n=0 \mathrm{LL}$ and at $v=7 / 3,8 / 3,10 / 3,11 / 3$ and $13 / 3$ in the $n=1 \mathrm{LL}$. Additionally, a weak minimum in $R_{x x}$ at $v=8 / 5$ is suggestive of an emerging FQHE state at this filling. With the exception of $1 / 3,2 / 3$ and $7 / 3$, all observed Hall conductivity plateaux are within $1 \%$ of their expected value. At $1 / 3$ and $2 / 3$, the Hall plateaux are ' $N$ ' shaped and do not exhibit exact quantization, typical of FQH states not yet fully formed in the magnetic field. The weak formation of the $1 / 3$ and $2 / 3$ states may be related to the presence of a competing, insulating phase at low density ${ }^{22}$ (see Supplementary Information).

The gap energies of the FQHE states were measured from the temperature dependence of the $R_{x x}$ minima in the thermally activated regime; $R_{x x} \propto e^{\Delta / 2 k_{\mathrm{B}} T}$, where $\Delta$ is the energy gap, $k_{\mathrm{B}}$ is Boltzmann's constant and $T$ is the electron temperature (Fig. 3). For all observed FQH states, the gaps are large in spite of considerable LL broadening due to disorder. For example, the measured gap at $v=4 / 3$ is $16 \mathrm{~K}$ at $35 \mathrm{~T}$. The large gaps probably result from the lower dielectric screening and near-zero width of the 2DES in comparison to conventional semiconductor heterostructures, both of which increase the effective strength of the electron interactions that give rise to the FQHE. Interestingly, the even-numerator and odd-numerator states exhibit opposite trends, with the even-numerator decreasing with increasing LL index, whereas the odd-numerator states actually grow in strength. Although the proximity of the $1 / 3$ state to the insulating state does not allow us to measure $\Delta_{1 / 3}$, recent measurements performed on multiterminal suspended graphene indicate that $\Delta_{1 / 3}$ is indeed larger than any gap values measured in this device ${ }^{23}$. The trend in the gap scaling with filling fraction may be a consequence of the relativistic dispersion relation, which modifies the electron interactions in graphene $e^{11,24}$. In the context of the composite fermion theory, the fact that the even-numerator gaps are greater than those of the odd numerator is analogous to the broken symmetry IQHE of real electrons, where the even-integer states emerge from the otherwise four-fold degenerate LLs with stronger gaps than the odd-integer states ${ }^{19}$.

Measurement of the even-numerator gaps over a range of perpendicular fields is shown in Fig. $3 \mathrm{~d}$. In the simplest picture of spinless, non-interacting composite fermions, the FQHE energy gaps are set by the Coulomb interaction between real electrons and therefore exhibit a $\sqrt{B}$-dependence with magnetic field. However, the near degeneracy of the real spin can give rise to spin-textured charged excitations, resulting in a nearly linear dependence of the FQHE gaps on magnetic field ${ }^{25}$. A question of fundamental interest is whether incompressible states at fractional filling in graphene support spin-textured excitations, and how these relate to the underlying symmetry of the ground state. The solid and dashed lines in Fig. 3d are attempts to fit the data using square root and linear $B$-dependences, respectively. Both fit the data equally well, making it impossible to distinguish them at present. We use the $y$ axis intercepts of the two fits to estimate the disorder-induced LL broadening to be in the range $\Gamma \sim 10-30 K$, giving an estimate for the intrinsic $4 / 3$ gap at $35 \mathrm{~T}$ of $30-50 \mathrm{~K}$, or $0.05-0.07 e^{2} / \epsilon l_{\mathrm{B}}$ in Coulomb energy units. Here we have taken the effective dielectric constant for graphene to be $\epsilon \sim 5$, calculated from the internal electron screening due to interband transitions ${ }^{26}$, where the external dielectric environment is taken as an average of vacuum $\left(\epsilon_{\mathrm{vac}}=1\right)$ and the underlying $\mathrm{BN}$ substrate $\left(\epsilon_{\mathrm{BN}} \sim 3.5\right)$.

In the $n=1 \mathrm{LL}$, the disorder limited $8 / 3$ gap measures approximately $6 \mathrm{~K}$. This is more than an order of magnitude larger gap than measured at the same total filling in GaAs devices ${ }^{27}$, where mobilities are in excess of $10^{7} \mathrm{~cm}^{2} \mathrm{~V}^{-1} \mathrm{~s}^{-1}$, and remains 2-3 

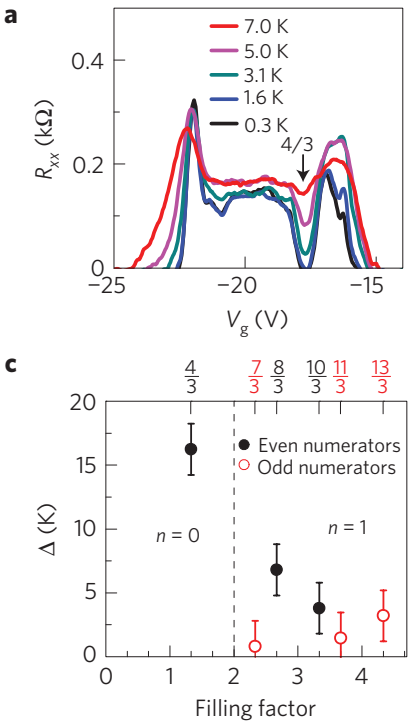

b

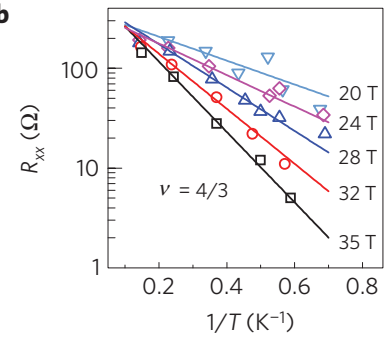

d

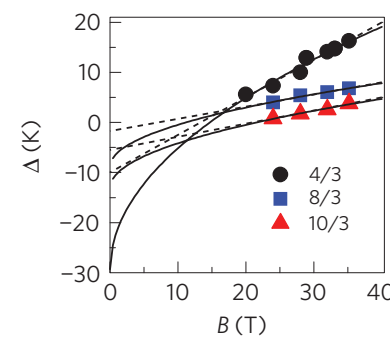

Figure 3 | Energy gaps. a, Magnetoresistance versus temperature near $v=4 / 3$ at $B=35 \mathrm{~T}$. $\mathbf{b}$, Arrhenius plot of the $R_{x x}$ minimum at $v=4 / 3$ for several magnetic fields. Energy gaps versus filling factor at $B=35 \mathrm{~T}$ and versus perpendicular magnetic field are shown in $\mathbf{c}$ and $\mathbf{d}$, respectively. Dashed and solid lines in $\mathbf{d}$ are fits assuming linear and square root $B$-dependences, respectively.

times larger even after accounting for the higher magnetic field at which our device was measured. This is consistent with the theoretical expectation that electron-electron interactions in the $n=1 \mathrm{LL}$ of graphene are expected to be similar in strength to the $n=0 \mathrm{LL}$ in GaAs (ref. 8).

No FQHE state appears in our samples at $v=5 / 3$. As a result of the Berry phase shift in graphene, the lowest LL for electrons (holes) begins at filling factor $v=-2(+2)$, in contrast to conventional zero-Berry phase materials, such as GaAs, in which the lowest LL begins at filling fraction $v=0$. Filling factor $\pm 5 / 3= \pm 2 \mp 1 / 3$ is thus expected to be the closest analogue to the $1 / 3$ state in conventional systems, making the non-observation of the $5 / 3$ state conspicuous given that the $1 / 3$ Laughlin state in conventional systems is generally robust. A distinguishing feature of graphene is the four-fold internal degeneracy, and in the presence of a large magnetic field we may consider three scenarios: (1) all degeneracies are lifted by coupling to external fields and FQHE states cannot mix spin/valley branches; (2) only one degeneracy, either spin or valley, is fully lifted, preserving an $\mathrm{SU}(2)$ symmetry in the remaining degenerate space and allowing, for example, mixed pseudospin but not mixed-spin FQH states; or (3) both Zeeman and valley splitting terms are sufficiently small that Coulomb interactions mix all branches, allowing mixed-spin/valley FQHE states and excitations. The remaining degeneracy should be reflected in the particle-hole symmetry of the FQHE states; in particular, we expect particle-hole conjugate FQH states within a single LL to have similar spin textures and gaps in the absence of any second order effects such as LL mixing ${ }^{28}$. The presence of the $1 / 3,2 / 3$ and $4 / 3$ together with the absence of the $5 / 3$ is inconsistent with both scenarios (1) and (2), which require simultaneous observation of either $4 / 3$ and $5 / 3$, or $1 / 3$ and $5 / 3$, respectively (Fig. $2 \mathrm{~d}$ ). The absence of any particle-hole symmetry except across the full $n=0 \mathrm{LL}$ is therefore consistent with the scenario where symmetry breaking terms remain sufficiently small that an approximate SU(4) symmetry is preserved. We note that previously studied multicomponent 2DES such as AlAs and strained silicon ${ }^{28,29}$, in addition to differences in the orbital character of the wavefunctions, differ from graphene in that the larger ratio between Zeeman and

Coulomb energies effectively freezes out the spin degree of freedom at large magnetic fields.

Whether an approximate $\mathrm{SU}(2)$ or $\mathrm{SU}(4)$ symmetry is assumed, a fully spin- and valley-polarized ground state with low-lying valley skyrmion excitations is theoretically expected at $v=5 / 3$ (refs $8,9,11,14,15)$. Theoretical calculations for a corresponding valley skyrmion gap $\left(0.03-0.043 e^{2} / \epsilon l_{\mathrm{B}}\right.$; refs 8,16$)$ are comparable to the $\sim 0.02-0.04 e^{2} / \epsilon l_{\mathrm{B}} \mathrm{LL}$ broadening estimated from our measurement of the $4 / 3$ gap, possibly explaining our nonobservation of the 5/3. At even-numerator filling in the lowest LL, such as at $8 / 5$ and $4 / 3$, a valley-unpolarized ground state is theoretically predicted ${ }^{8,11,14,16}$, with larger energy excitations than those occurring at $5 / 3$, qualitatively consistent with our experimental findings. The smallness of the Zeeman energy in comparison with Coulomb energy theoretically allows for the possibility ${ }^{17}$ of real spin-reversed excitations at $v=1 / 3$, and may account for the relative strength of the observed $1 / 3$ in comparison with the absent $5 / 3$. Alternatively, disorder may have a different effect near the edge of the LL (5/3) than it does near the insulating state at charge neutrality (1/3), possibly accounting for some of the observed difference. Further experimental and theoretical efforts in determining the real- and valley-spin texture of the FQHE ground states and their elementary excitations are necessary to resolve this puzzle.

The second LL (SLL) shows nearly all multiples of $1 / 3$ within the experimental range. For example, the $7 / 3=2+(1 / 3)$ state, the SLL analogue of the $5 / 3$, is present, and the $11 / 3=2+(5 / 3)$ and $10 / 3=2+(4 / 3)$, analogues of the $1 / 3$ and $2 / 3$, are also both well developed. Observation of the FQHE at $v=13 / 3$ represents, to our knowledge, the first clear measurement of the FQHE at a filling factor $v>4$. This may be attributed at least in part to the fact that the FQHE states between $4<v<6$ continue to belong to the $n=1 \mathrm{LL}$ because of the four-fold symmetry of single-particle LLs in graphene.

\section{Methods}

To fabricate the graphene-on-h-BN device a similar mechanical transfer technique to that described in ref. 18 was used but with the water-soluble sacrificial layer replaced by a polyvinyl alcohol (PVA) layer. This allowed mechanical peeling of the PMMA membrane without the need to expose the graphene/PMMA substrate to a water bath, thereby achieving a fully dry transfer method. Electrical leads consisting of a $\mathrm{Cr} / \mathrm{Au}$ metal stack were deposited using standard electron-beam lithography, after which the sample was etched into an approximately square Hall bar by exposure to oxygen plasma (Fig. 1b). Four-terminal transport measurements were performed using a lock-in amplifier at $17 \mathrm{~Hz}$ with a $10 \mathrm{nA}$ source current. The sample was measured in a $35 \mathrm{~T}$ resistive magnet and ${ }^{3} \mathrm{He}$ cryostat (sample in vapour). We note that there is an apparent asymmetry in the transport, with p-type carriers showing better developed quantum Hall effect features/higher mobility than n-type carriers, particularly in the second LL. This higher mobility for p-type carriers is not a systematic trend, as we have observed higher mobility for $\mathrm{n}$-type carriers in other samples. Therefore, although data acquired at negative gate voltages (p-type carriers) is primarily discussed, we expect our results should be consistent with samples where $n$-type carriers experience comparable disorder.

Received 19 October 2010; accepted 21 April 2011; published online 22 May 2011

\section{References}

1. Tsui, D. C., Stormer, H. L. \& Gossard, A. C. Two-dimensional magnetotransport in the extreme quantum limit. Phys. Rev. Lett. 48, 1559-1562 (1982).

2. Laughlin, R. B. Anomalous quantum Hall effect: An incompressible quantum fluid with fractionally charged excitations. Phys. Rev. Lett. 50, 1395-1398 (1983).

3. Halperin, B. Theory of the quantized Hall conductance. Helv. Phys. Acta 56, 75-102 (1983).

4. Jain, J. K. Composite-fermion approach for the fractional quantum Hall effect. Phys. Rev. Lett. 63, 199-202 (1989).

5. Das Sarma, S. \& Pinczuk, A. Perspectives in Quantum Hall Effects (Wiley, 1997).

6. Novoselov, K. et al. Two-dimensional gas of massless Dirac fermions in graphene. Nature 438, 197-200 (2005). 
7. Zhang, Y., Tan, Y., Stormer, H. \& Kim, P. Experimental observation of the quantum Hall effect and Berry's phase in graphene. Nature 438, 201-204 (2005).

8. Toke, C., Lammert, P. E., Crespi, V. H. \& Jain, J. K. Fractional quantum Hall effect in graphene. Phys. Rev. B 74, 235417 (2006).

9. Yang, K., Das Sarma, S. \& MacDonald, A. H. Collective modes and skyrmion excitations in graphene SU(4) quantum Hall ferromagnets. Phys. Rev. B 74, 075423 (2006).

10. Nomura, K. \& MacDonald, A. H. Quantum Hall ferromagnetism in graphene. Phys. Rev. Lett. 96, 256602 (2006).

11. Apalkov, V. M. \& Chakraborty, T. Fractional quantum Hall states of Dirac electrons in graphene. Phys. Rev. Lett. 97, 126801 (2006).

12. Goerbig, M. O. \& Regnault, N. Analysis of a SU(4) generalization of Halperin's wave function as an approach towards a SU(4) fractional quantum Hall effect in graphene sheets. Phys. Rev. B 75, 241405 (2007).

13. Khveshchenko, D. V. Composite Dirac fermions in graphene. Phys. Rev. B 75 153405 (2007)

14. Toke, C. \& Jain, J. K. SU(4) composite fermions in graphene: Fractional quantum Hall states without analog in GaAs. Phys. Rev. B 75, 245440 (2007).

15. Shibata, N. \& Nomura, K. Coupled charge and valley excitations in graphene quantum Hall ferromagnets. Phys. Rev. B 77, 235426 (2008).

16. Shibata, N. \& Nomura, K. Fractional quantum Hall effects in graphene and its bilayer. J. Phys. Soc. Jpn 78, 104708 (2009).

17. Papić, Z., Goerbig, M. O. \& Regnault, N. Atypical fractional quantum Hall effect in graphene at filling factor 1/3. Phys. Rev. Lett. 105, 176802 (2010).

18. Dean, C. R. et al. Boron nitride substrates for high-quality graphene electronics. Nature Nanotech. 5, 722-726 (2010).

19. Zhang, Y. et al. Landau-level splitting in graphene in high magnetic fields. Phys. Rev. Lett. 96, 136806 (2006)

20. Du, X., Skachko, I., Duerr, F., Luican, A. \& Andrei, E. Y. Fractional quantum Hall effect and insulating phase of Dirac electrons in graphene. Nature 462, 192-195 (2009).

21. Bolotin, K. I., Ghahari, F., Shulman, M. D., Stormer, H. L. \& Kim, P. Observation of the fractional quantum Hall effect in graphene. Nature 462, 196-199 (2009).

22. Checkelsky, J. G., Li, L. \& Ong, N. P. Zero-energy state in graphene in a high magnetic field. Phys. Rev. Lett. 100, 206801 (2008).

23. Ghahari, F., Zhao, Y., Cadden-Zimansky, P., Bolotin, K. \& Kim, P. Measurement of the $v=1 / 3$ fractional quantum Hall energy gap in suspended graphene. Phys. Rev. Lett. 106, 046801 (2011).
24. Goerbig, M. O., Moessner, R. \& Douçot, B. Electron interactions in graphene in a strong magnetic field. Phys. Rev. B 74, 161407 (2006).

25. Dethlefsen, A. F., Mariani, E., Tranitz, H-P., Wegscheider, W. \& Haug, R. J. Signatures of spin in the $v=1 / 3$ fractional quantum Hall effect. Phys. Rev. B 74, 165325 (2006).

26. Ando, T. Screening effect and impurity scattering in monolayer graphene. J. Phys. Soc. Jpn 75, 074716 (2006).

27. Kumar, A., Manfra, M. J., Csáthy, G. A., Pfeiffer, L. N. \& West, K. W. Nonconventional odd denominator fractional quantum Hall states in the second Landau level. Phys. Rev. Lett. 105, 246808 (2010).

28. Padmanabhan, M., Gokmen, T. \& Shayegan, M. Composite fermion valley polarization energies: Evidence for particle-hole asymmetry. Phys. Rev. B 81, 113301 (2010).

29. Lai, K., Pan, W., Tsui, D. C. \& Xie, Y-H. Fractional quantum Hall effect at $v=2 / 3$ and $4 / 3$ in strained Si quantum wells. Phys. Rev. B 69, 125337 (2004)

\section{Acknowledgements}

We thank J. K. Jain, C. Toke, N. Shibata, M. O. Goerbig and M. Foster for discussions, J. Sanchez-Yamagishi and P. Jarillo-Herrero for fabrication advice regarding the PVA, and I. Meric, Z. Kagan, A. Tsoi, N. Baklitskaya and I. Mendonca for help with the device preparation. A portion of this work was performed at the National High Magnetic Field Laboratory, which is supported by National Science Foundation Cooperative Agreement No. DMR-0654118, the State of Florida and the US Department of Energy. This work is supported by DARPA CERA, AFOSR MURI, FCRP through C2S2 and FENA, NSEC (No. CHE-0117752) and NYSTAR. P.K. and A.F.Y. acknowledge support from DOE (DE-FG02-05ER46215).

\section{Author contributions}

C.R.D. and A.F.Y. performed all experiments including sample fabrication and measurement, and wrote the paper. P.C-Z. contributed to sample measurement. L.W. and H.R. contributed to sample fabrication. K.W. and T.T. synthesized the h-BN samples. J.H., P.K. and K.L.S. advised on experiments.

\section{Additional information}

The authors declare no competing financial interests. Supplementary information accompanies this paper on www.nature.com/naturephysics. Reprints and permissions information is available online at http://www.nature.com/reprints. Correspondence and requests for materials should be addressed to P.K. 